\title{
STRUCTURAL DESIGN FOR OBTAINING DESIRED EIGENFREQUENCIES BY USING THE TOPOLOGY AND SHAPE OPTIMIZATION METHOD ${ }^{\dagger}$
}

\author{
Zheng-Dong Ma, Hsien-Chie Cheng and Noboru Kikuchi \\ Department of Mechanical Engineering and Applied Mechanics, The University of Michigan, \\ Ann Arbor, MI 48109-2215, U.S.A.
}

\begin{abstract}
A topology and shape optimization method is presented for structural eigenfrequency optimization problems using the concept of Optimal Material Distribution (OMD). First. a mean-eigenvalue corresponding to the multiple eigenfrequencies of a structure is defined. Three optimization problems are then considered for obtaining the desired eigenfrequencies using this mean-eigenvalue: maximization of the specified structural eigenfrequencies, maximization of the distances of the specified structural eigenfrequencies from a given frequency or frequencies, and optimization of the structure for obtaining prescribed eigenfrequencies. Several examples are presented to demonstrate the capability of this new technique which can be used to deal with a wide range of practical design problems for improving the dynamic nature of a structure.
\end{abstract}

\section{NOMENCLATURE}

$a\left(a_{e}\right) \quad$ design variable of the microstructure (element)

$b\left(b_{t^{\prime}}\right) \quad$ design variable of the micro structure (element)

B $_{e} \quad$ strain shape function of a finite element

$\mathbf{D}^{h} \quad$ homogenized elasticity matrix without rotation

D. $\quad$ rotated homogenized elasticity matrix with respect to element $e$

$\mathbf{k}_{e}, \mathbf{K}$ stiffness matrix (element/global)

$\mathbf{m}_{e}, \mathbf{M}$ mass matrix (element/global)

$\mathbf{N}_{c} \quad$ shape function of a finite element

T, rotation matrix

$w_{i} \quad$ weighting function

$x_{i}^{k} \quad i$ th design variable in the $k$ th iteration

$\rho \quad$ the mass density

$\boldsymbol{\rho}^{h} \quad$ homogenized density matrix without rotation

$\boldsymbol{\rho}_{e} \quad$ shift homogenized density matrix with respect to element $e$

$\mu^{k} \quad$ shift parameter in the $k$ th iteration [Eqs (22) and (23)]

$\eta \quad$ given power parameter [Eq. (22)]

$\lambda$ Lagrange multiplier

$\lambda_{n} \quad n$th eigenvalue of a structure

$\lambda^{*} \quad$ mean eigenvalue

$\lambda_{0}^{*}, \lambda_{0,}$ parameters in the mean eigenvalue [Eq.(5)]

$\phi_{n} \quad n$th eigenvector of a structure

$\phi_{n_{1, k}} \quad$ components of the $n_{i}$ th eigenvector with respect to the $e$ th element

$\theta\left(\theta_{c}\right)$ orientation of microstructure (with respect to element $e$ )

$\Omega \quad$ structural domain

$\Omega_{e} \quad$ domain of finite element $e$

\section{INTRODUCTION}

Despite that structural optimum design problems have been extensively studied in the last few

†Presented at the Second U.S. National Congress on Computational Mechanics, 16-18 August 1993, Washington. D.C. decades, ${ }^{13}$ there are few works on the subject of structural dynamic optimization. Most of these efforts are concentrated on optimization problems with dynamic constraints, ${ }^{4}{ }^{6}$ but not problems with treat the dynamic nature of a structure as the subject in the optimization process. In many cases, however, modifying a structure to maximally improve the dynamic behavior of the structure is very important. For example, in a car body design, it is a very important to minimize the vibration and noise under known design constraints and loading conditions for developing a high-quality automobile. Another example is in the design of space vehicles. In this case it is highly desirable to optimize several structural eigenfrequencies for the desired values in order to avoid coupling with the control system. ${ }^{7}$ In these cases, the dynamic behavior of a system should be treated as an object of the optimization process instead of dealing with it as a constraint, as most research does.

Niordson ${ }^{8}$ opened the field of the eigenfrequency optimization. He considered a problem of maximizing the fundamental eigenfrequency of a vibrating beam with a specified volume constraint. Brach extended Niordson's work by considering other boundary conditions. Prager and Taylor ${ }^{10}$ presented a necessary and sufficient condition for maximizing fundamental eigenfrequency of sandwich structures. Zarghamee ${ }^{11}$ reported a discrete problem of the maximum eigenfrequency type using a gradient projection method. Prato ${ }^{12}$ then improved the computational scheme for this class of problems. Olhoff ${ }^{13}$ presented results for maximizing a higher-order eigenfrequency subject to a given volume constraint. Bendsøe and Olhoff ${ }^{14}$ also considered the problem of maximizing the difference between two adjacent eigenfrequencies 
of a beam. An extensive review paper was given by Olhoff ${ }^{15}$ about this field.

Even though the use of previous techniques developed for sizing optimization problems has made it possible to obtain the optimal structure for some particular eigenfrequency optimization problems, it is still difficult to obtain the desired structural eigenfrequencies because only a limited modification can be made by changing the structural sizes. Thus, it is necessary to consider modifying both the topology and shape for a truly optimal design. Bendsøe and Kikuchi ${ }^{16}$ presented a method based on the concept of Optimal Material Distribution (OMD) which transfers the structural optimization problem to the problem of finding the OMD with a specified design domain. This idea has opened up a new class of structural optimization problems, and it has been successfully applied to the cases for structural stiffness, ${ }^{16}$ structural eigenfrequency, ${ }^{17.18}$ and frequency response problems. ${ }^{19}$

There are three kinds of optimization problems which can be considered for the eigenfrequency optimization of a structural system. The first one is to maximize the specified structural eigenfrequencies, the second is to maximize the distances of the specified structural eigenfrequencies from a given frequency or frequencies, and the third is to optimize a structure for obtaining prescribed desired eigenfrequencies. It is noted that previous research dealt with only the first kind of problem except Bendsøe and Olhoff, ${ }^{14}$ which studied a problem of the second kind for very simple structural models. Furthermore, only changing of structural sizes such as the radius of the cross-section of a beam has been considered in the previous work.

In order to solve a general eigenfrequency optimization problem, a multi-eigenvalue optimization technique is presented with the use of the topology and shape optimization method in this work. First, a mean-eigenvalue is proposed as a combination of multiple eigenvalue to define a mathematical formulation of the optimization problem, and three new objective functions are then proposed for obtaining desired structural eigenfrequencies using the meaneigenvalue. It will be shown that the use of the new technique presented in this paper can greatly improve the solution of eigenfrequency optimization problems, and can be applied to a wide range of practical problems to improve the dynamic behavior of a structure.

\section{EIGENFREQUENCY OPTIMIZATION PROBLEMS}

\subsection{State equation}

Using the finite element method the state equation for the natural frequencies of a linearly elastic structure can be written as follows:

$$
\left(\mathbf{K}-\lambda_{n} \mathbf{M}\right) \phi_{n}=0,
$$

where, $\lambda_{n}$ stands for the $n$th eigenvalue, $\phi_{n}$ its corresponding eigenvevctor, $\mathbf{M}$ the mass matrix and $\mathbf{K}$ the stiffness matrix defined by:

$$
\mathbf{K}=\underset{e=1}{n_{e l}} \mathbf{k}_{e}, \quad \mathbf{M}=\underset{e=1}{\stackrel{n_{e l}}{A} \mathbf{m}_{e},}
$$

respectively, where $A_{e=1}^{n_{e l}}$ stands for the finite element element assembly operator. Here $\mathbf{k}_{e}$ and $\mathbf{m}_{e}$ are the element stiffness and mass matrices obtained by

$$
\mathbf{k}_{e}=\int_{\Omega_{e}} \boldsymbol{B}_{e}^{T} D_{e} \boldsymbol{B}_{e} \mathrm{~d} \boldsymbol{\Omega}, \quad \mathbf{m}_{e}=\int_{\Omega_{e}} \mathbf{N}_{e}^{T} \boldsymbol{\rho}_{e} \mathbf{N}_{e} \mathrm{~d} \mathbf{\Omega},
$$

where $\mathbf{D}_{e}$ and $\boldsymbol{\rho}_{e}$ stand for the homogenized elasticity and density with respect to the element $e$, respectively, $\mathbf{B}_{e}$ the strain shape function, $\mathbf{N}_{e}$ the displacement shape function.

\subsection{Mean eigenvalue}

Maximizing a chosen eigenfrequency of a structure in the structural eigenfrequency optimization problem has been extensively discussed. ${ }^{13}$ However, in a vibrating system, there may be several excitation frequencies distributed in the different frequency domains. In order to avoid these excitation frequencies, we may need to deal with not only a single eigenfrequency of the structure, but also several chosen eigenfrequencies. Even in the case of maximizing a single eigenfrequency of a structure, it is necessary to consider multiple eigenfrequencies as the projective function in the optimization process. As previously discussed, ${ }^{18}$ for example, in topology and shape optimization problems, large changes in structural eigenfrequencies may cause structural eigenfrequencies to switch their orders within the optimization process. In this case, if we only use a single eigenfrequency as the objective function, the sensitivities of the objective function may be discontinuous, and oscillation and divergence may be caused in the optimization process. In order to overcome this problem, a specified multi-frequency objective function was suggested as follows: ${ }^{18}$

$$
\lambda^{*}=\lambda_{0}+\sum_{i=1}^{m} w_{i}\left(\sum_{i=1}^{m} \frac{w_{i}}{\lambda_{n_{i}}-\lambda_{0}}\right)^{-1},
$$

where, $\lambda_{n_{i}}\left(n_{i}=n_{1}, n_{2}, \ldots, n_{m}\right)$ stand for the specified eigenvalues, while $\lambda_{n_{i}}$ is the $n_{i}$ th eigenvalue $(i=1,2, \ldots, m)$ (noting here, orders $n_{1}, n_{2}, \ldots, n_{m}$ can be discontinuous integers, e.g. we can choose $n_{1}=2, n_{2}=5$, etc. $) . w_{i}(i=1,2, \ldots, m)$ stands for given weighting coefficients, and $\lambda_{0} \geqslant 0$ a given parameter. It was still considered that the major purpose of the optimization is to maximize a single eigenvalue. As shown in Eq. (4), the eigenvalue which is closest to the parameter $\lambda_{0}$ has the greatest contribution to the objective function $\lambda^{*}$ assuming all weighting coefficients are the same, therefore this eigenvalue will be the major object in the optimization process. In a 
special case, if choose $\lambda_{0}=0$, then the smallest eigenvalue in $\lambda_{n_{i}}\left(n_{i}=n_{1}, n_{2}, \ldots, n_{m}\right)$ will be the major object in the optimization process, i.e. it will experience the largest change in the optimization process. Since when two modes whose eigenvalues are in Eq. (4) switch their orders within the optimization process, the change in the objective function will be smoother than in the case when one of these eigenvalues is not in $\lambda_{n_{i}}\left(n_{i}=n_{1}, n_{2}, \ldots, n_{m}\right)$. Therefore, objective function Eq. (4) can improve the solution comparing with the use of only one single eigenvalue.

As shown in Eq. (4), if assuming $m=1$, Eq. (4) is reduced to $\lambda^{*}=\lambda_{n_{t}}$, and the corresponding optimization problem becomes identical to that of optimizing a single eigenfrequency. In this work, we shall call $\lambda^{*}$ as the mean-eigenvalue. In addition, in order to consider more general optimization problems, we extend the mean-eigenvalue to the following form: eigenfrequency optimization problems mentioned before can be defined by the mean-eigenvalue: (1) maximizing the specified structural eigenfrequencies; (2) maximizing the distances between the specified structural eigenfrequencies and a given frequency or frequencies; and (3) optimizing the structure for obtaining the prescribed desired eigenfrequencies.

Eigenfrequency optimization problem 1 . If we choose power $n$ as an odd number (note that if power $n$ is an odd number, then the increase of any eigenvalue in the objective function $\lambda^{*}$ will result in the increase of $\lambda^{*}$ ), and define the optimization problem as:

$$
\text { Maximize } \dot{\lambda}^{*} \text {, }
$$

the eigenvalues $\lambda_{n_{i}}\left(n_{i}=n_{1}, n_{2}, \ldots, n_{m}\right)$, which are in the mean-eigenvalue, will be maximized in the

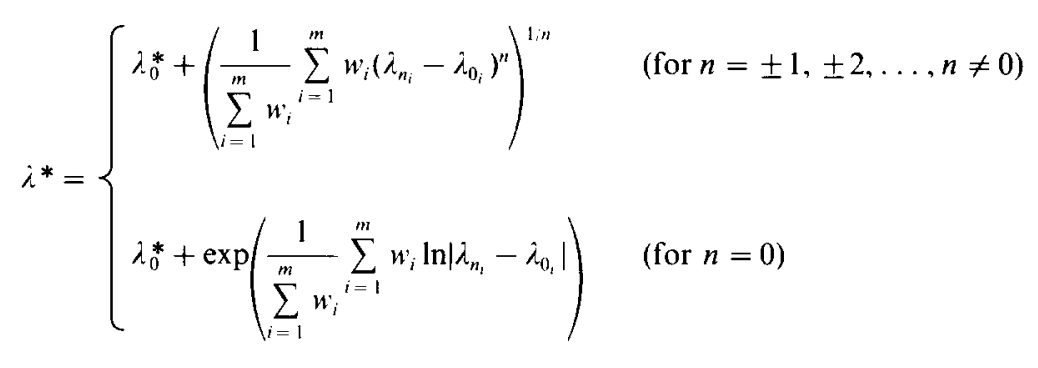

where, $n$ is a given power and $\lambda_{0}^{*}$ and $\lambda_{0_{i}}(i=1,2, \ldots, m)$ are the given parameters.

As shown in Eq. (5), when $n \geqslant 0$ the eigenvalue (assume $\lambda_{n_{i}}$ ) which is the farthest from the corresponding given parameter $\hat{i}_{0_{i}}$ has the largest contribution to the mean-eigenvalue (assuming that all weighting coefficients $w_{i}(i=1,2, \ldots, m)$ are the same). Conversely, if $n<0$, the eigenvalue which is the closest to the corresponding given parameter has the largest contribution to the mean-eigenvalue. Therefore, if use the mean-eigenvalue as the objective function, the eigenvalue which is the closest to (when $n<0$ ) or the farthest from (when $n \geqslant 0$ ) the corresponding given parameter will be the major object in the optimization problem. In a special case, if let $\lambda_{0_{t}}=0(i=1,2, \ldots, m)$, the highest (when $n \geqslant 0$ ) or lowest (when $n<0$ ) eigenvalue in $\lambda_{n_{i}}\left(n_{i}=\right.$ $n_{1}, n_{2}, \ldots, n_{m}$ ) will have the largest contribution to the mean-eigenvalue, and then it becomes the major object of the optimization while the rest would make very minor contribution to the objective function.

Note that proper choice of the weighting coefficients $w_{i}(i=1,2, \ldots, m)$ can yield an increase or reduction of the contribution of eigenvalues $\lambda_{n_{i}}\left(n_{i}=n_{1}, n_{2}, \ldots, n_{m}\right)$, and then we can make a desired multi-eigenfrequency optimization.

\subsection{Objective functions using the mean-eigenvalue}

Choosing different values of parameters $n$ and $\lambda_{0_{i}}(i=1,2, \ldots, m)$ of Eq. (5), all three kinds of sense of the weighted average of the reciprocals of the eigenvalues. Here, parameters $\lambda_{0}^{*}$ and $\lambda_{0_{i}}(i=1,2, \ldots, m)$ can be specified as any values, for example, zero or a given constant. Assuming all weighting coefficients $w_{i}(i=1,2, \ldots, m)$ are the same, the eigenvalue which is the closest to (when $n<0$ ) or the farthest from (when $n \geqslant 0$ ) the corresponding given parameter $\lambda_{0_{i}}$ will be subject to the largest increase in the optimization process.

Eigenfrequency optimization problem 2 . In order to maximize the distances among specified structural eigenfrequencies and a given frequency or frequencies, we can choose $n$ as a negative even number, e.g. $n=-2$, and $w_{0_{i}}(i=1,2, \ldots, m)$ as the given frequencies, while $\lambda_{0_{i}}=\left(2 \pi \omega_{0_{i}}\right)^{2}$. (Note that if power $n$ is chosen as an even number, then the increase (decrease) of any distance $\left|\lambda_{n_{i}}-\lambda_{0_{i}}\right|$ included in the objective function $\lambda^{*}$ will result in the increase (decrease of $\lambda^{*}$ ). The optimization problem is then defined as:

\section{Maximize $\lambda^{*}$.}

In this case, distances $\left|\lambda_{n_{i}}-\lambda_{0_{i}}\right|(i=1,2, \ldots, m)$ will be maximized in the sense of the weighted average.

Note that if power $n$ is chosen to be a negative even number, then the eigenfrequency that is the closest to the given frequency would be away from this one with the fastest speed. 
Eigenfrequency optimization problem 3 . In order to obtain prescribed eigenfrequencies desired for a structure, we can choose $n$ to be positive even number, e.g. $n=2$, and $\omega_{0 i}\left(\lambda_{0_{i}}=\left(2 \pi \omega_{0_{i}}\right)^{2} ; i=1,2, \ldots, m\right)$ as desired eigenfrequencies, while the optimization problem is defined as:

\section{Minimize $\lambda^{*}$.}

Then, distances between the structural eigenfrequencies and the desired ones, i.e. $\left|\lambda_{n_{i}}-\lambda_{0_{i}}\right|$ $(i=1,2, \ldots, m)$, will be minimized in the optimization process.

Note that if we choose $n$ as a positive even number, the eigenfrequency which is the most away from its desired value will approach to its desired value with the fastest speed.

\section{STRUCTURAL MODEL USING A MICROSTRUCTURE}

The topology and shape optimization technique proposed by Bendsøe and Kikuchi ${ }^{16}$ is employed for solving the problems defined in above. To simplify the problem, we shall only consider plane-stress problems, and a prescribed admissible structural domain that is filled by variable microstructures. Figure 1 shows a microstructure assumed in the present work that contains an empty rectangle inside a unit cell in a structural domain. The inside cavity of the unit cell is varying in the design domain along with the design variables, $a$ and $b$. The microstructure becomes a complete void when $a=b=0$ and a complete solid when $a=b=1$. The unit cell is also rotated by the orientation $\theta$ from a given $x-y$ coordinate system as shown in Fig. 1 to consider preference of the possibly orthotropic material principle axes.

Because of existence of the micro-cavities, the elasticity and density are non-homogeneous in the

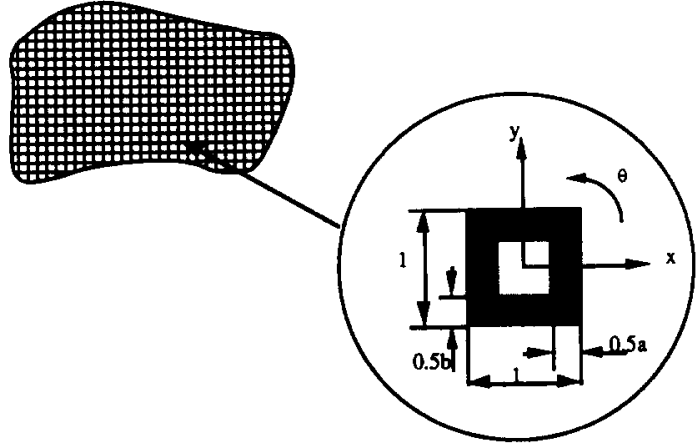

Fig. 1. The microstructure in a structural domain.

using the finite element method for the averaged (i.e. homogenized) problem, we can further assume that in an individual finite element, the microstructures are constant together with the sizes and rotation, $a=a_{e}, b=b_{e}$ and $\theta=\theta_{e}$ in element $e$, where $a_{e}, b_{e}$ and $\theta_{e}$ stand for the discrete design variables assigned in finite element $e$ which are finite element approximations of the distributed design variables $a$, $b$ and $\theta$. Thus, the homogenized elasticity and density in element $e$ are the functions of $a_{e}, b_{e}$ and $\theta_{e}$.

Without considering rotation of the microstructure, i.e. with $\theta_{e}=0$, the homogenized elasticity and density of a microstructure may be computed as:

$$
\mathbf{D}^{h}=\left[\begin{array}{ccc}
D_{11}^{h} & D_{12}^{h} & 0 \\
D_{12}^{h} & D_{22}^{h} & 0 \\
0 & 0 & D_{33}^{h}
\end{array}\right]=\mathbf{D}^{h}\left(a_{e}, b_{e}\right)
$$

and

$$
\boldsymbol{\rho}^{h}=\boldsymbol{\rho}^{h}\left(a_{e}, b_{e}\right)
$$

where $\mathbf{D}^{h}$ stands for the homogenized elasticity and $\boldsymbol{\rho}^{h}$ the homogenized density.

The homogenized elasticity is then rotated by the rotation matrix,

$$
\mathbf{T}_{e}=\left[\begin{array}{ccc}
\cos ^{2} \theta_{e} & \sin ^{2} \theta_{e} & \cos \theta_{e} \sin \theta_{e} \\
\sin ^{2} \theta_{e} & \cos ^{2} \theta_{e} & -\cos \theta_{e} \sin \theta_{e} \\
-2 \cos \theta_{e} \sin \theta_{e} & 2 \cos \theta_{e} \sin \theta_{e} & \cos ^{2} \theta_{e}-\sin ^{2} \theta_{e}
\end{array}\right],
$$

structural domain. Thus, the state equations cannot be solved in the closed form using a conventional analysis method. Furthermore, if the size of cavities are asumed to be finite, it becomes considerably difficult to analyse them by conventional finite element methods. Thus, it is assumed that the microstructures are infinitely small, and in an arbitrary point of the structural domain, around the point there are infinitely many repeated microstructures, i.e. the sizes $a, b$ and the orientation $\theta$ can be defined at an arbitrary point in the design domain. Under this assumption, the homogenization method can be utilized to define an average model for solving state equations which are characterized by the continuous homogenized elasticity and density. Furthermore, for a rotated microstructure. Then we have:

$$
\mathbf{D}_{e}=\mathbf{T}_{e}^{T} \mathbf{D}^{h} \mathbf{T}_{e}, \quad \boldsymbol{\rho}_{e}=\boldsymbol{\rho}^{h} .
$$

Since the density is a scalar function, we have the same for the rotated and unrotated microstructure.

Using $a_{e}, b_{e}$ and $\theta_{e}\left(e=1,2, \ldots, n_{e l}\right)$ as the design variables, the structural optimization problem is then conceived to an Optimal Material Distribution (OMD) problem. In the optimization process, the microstructures are varying between empty and solid. Thus, if the total amount of the material of the structure is assumed to be constant, then the pieces of the material are moved from a part of the structure 
to another while the optimization process is progressed. By moving the material to optimize the object function, we can obtain an OMD that define the optimal structure.

It should be noted that even though the orientational variable $\theta$ does not change the amount of material of a structure, it plays a very important role in the optimization process. Without rotation of the microstructure, the optimization process may not converge to an optimal structure..$^{20}$

\section{SENSITIVITIES OF THE MEAN-EIGENVALUE}

\subsection{Sensitivity for a size design variable}

The sensitivity of the mean-eigenvalue with respect to a size design variable, $a_{e}$ or $b_{e}$, can be obtained as
The sensitivity of the mean-eigenvalue with respect to an orientational design variable can be rewritten as: ${ }^{20}$

$$
\begin{aligned}
\frac{\partial \lambda^{*}}{\partial \theta_{e}}=2\left(g_{e}^{1} \cos 2 \theta_{e}\right. & -g_{e}^{2} \sin 2 \theta_{e} \\
& \left.+g_{e}^{3} \cos 4 \theta_{e}-g_{e}^{4} \sin 4 \theta_{e^{\prime}}\right),
\end{aligned}
$$

where, coefficients $g_{e}^{k}(k=1,2,3,4)$ are independent of $\theta_{e}$.

\section{CONSTRAINT FUNCTION AND ITS SENSITIVITIES}

It is assumed that the total mass of a structure is constrained by

$$
\int_{\Omega} \rho_{e} \mathrm{~d} \Omega \leqslant V_{0}
$$

$$
\frac{\partial \lambda^{*}}{\partial x}= \begin{cases}\frac{\left(\lambda^{*}-\lambda_{0}^{*}\right)^{1-n}}{\sum_{i=1}^{m} w_{i}} \sum_{i=1}^{m} \frac{w_{i}}{\left(\lambda_{n_{i}}-\lambda_{0_{i}}\right)^{1-n}} \phi_{n_{i}, e}^{T}\left(\frac{\partial \mathbf{k}_{e}}{\partial x}-\lambda_{n_{i}} \frac{\partial \mathbf{m}_{e}}{\partial x}\right) \phi_{n_{i}, e} & (\text { for } n= \pm 1, \pm 2, \ldots ; n \neq 0), \\ \frac{\left|\lambda^{*}-\lambda_{0}^{*}\right|}{\sum_{i=1}^{m} w_{i}} \sum_{i=1}^{m} \frac{w_{i}}{\left|\lambda_{n_{i}}-\lambda_{0_{e}}\right|} \phi_{n_{i}, e}^{T}\left(\frac{\partial \mathbf{k}_{e}}{\partial x}-\lambda_{n_{i}} \frac{\partial \mathbf{m}_{e}}{\partial x}\right) \phi_{n_{i}, e} & (\text { for } n=0)\end{cases}
$$

where, $x$ stands for a design variable $a_{e}$ or $b_{e}$ that belongs to element $e, \phi_{n_{i}, e}$ the components of the $n_{i}$ th eigenvector with respect to the $e$ th element, while $\partial \mathbf{k}_{\boldsymbol{e}} / \partial x$ and $\partial \mathbf{m}_{\varepsilon} / \partial x$ are calculated by

$$
\begin{gathered}
\frac{\partial \mathbf{k}_{e}}{\partial x}=\int_{\Omega_{e}} \mathbf{B}_{e}^{T} \frac{\partial \mathbf{D}_{e}}{\partial x} \mathbf{B}_{e} \mathrm{~d} \boldsymbol{\Omega}, \\
\frac{\partial \mathbf{m}_{e}}{\partial x}=\int_{\boldsymbol{\Omega}_{c}} \mathbf{N}_{e}^{T} \frac{\partial \boldsymbol{\rho}_{e}}{\partial x} \mathbf{N}_{e} \mathrm{~d} \boldsymbol{\Omega} .
\end{gathered}
$$

4.2. Sensitivity for an orientational design variable

For an orientational design variable $\theta_{e}$, the sensitivity of the mean-eigenvalue can be obtained as

$$
\frac{\partial \lambda^{*}}{\partial \theta_{e}}= \begin{cases}\frac{\left(\lambda^{*}-\lambda_{0}^{*}\right)^{1-n}}{\sum_{i=1}^{m} W_{i}} \sum_{i=1}^{m} \frac{w_{i}}{\left(\lambda_{n_{i}}-\lambda_{0_{i}}\right)^{1-n}} \phi_{n_{i}, e}^{T} \frac{\partial \mathbf{k}_{e}}{\partial \theta_{e}} \phi_{n_{i}, e} & (\text { for } n= \pm 1, \pm 2, \ldots ; n \neq 0), \\ \frac{\left|\lambda^{*}-\lambda_{0}^{*}\right|}{\sum_{i=1}^{m} w_{i}} \sum_{i=1}^{m} \frac{w_{i}}{\left|\lambda_{n_{i}}-\lambda_{0_{i}}\right|} \phi_{n_{i}, e}^{T} \frac{\partial \mathbf{k}_{e}}{\partial \theta_{e}} \phi_{n_{i}, e} & (\text { for } n=0),\end{cases}
$$

where

and

$$
\frac{\partial \mathbf{k}_{e}}{\partial \theta_{e}}=\int_{\Omega_{c}} \mathbf{B}_{e}^{T} \frac{\partial \mathbf{D}_{e}}{\partial \theta_{e}} \mathbf{B}_{e} \mathrm{~d} \boldsymbol{\Omega} .
$$

where $V_{0}$ is the given maximal total mass of the structure. In the present formulation with the microstructure shown in Fig. 1, we have $\rho_{e}=\rho_{0}\left(a_{e}+b_{e}-a_{e} b_{e}\right)$, and then the constraint function becomes

$$
h\left(a_{e}, b_{e}\right)=\sum_{e=1}^{n_{e l}} \rho_{0} \Omega_{e}\left(a_{e}+b_{e}-a_{e} b_{e}\right)-V_{0} \leqslant 0 .
$$

The sensitivities of the constraint function with respect to the design variables $a_{\varepsilon}$ and $b_{\varepsilon}$ can be obtained as

$$
\frac{\partial h}{\partial a_{e}}=\rho_{0} \Omega_{e}\left(1-b_{e}\right)
$$




\section{UPDATING ALGORITHM}

\subsection{Updating the size design variables}

An improved optimization algorithm, the Modified Optimality Criteria method (MOC), has been presented ${ }^{19}$ for updating the size variables in the topology and shape optimization problem of a vibrating structure. The basic idea is to apply a convex generalized-linearization approach using the shift parameter which corresponds to the Lagrange multiplier. The dual method is also employed to separate a multi-variable minimization problem to a series of one-dimensional problems. The updating rule proposed is:

$$
\begin{aligned}
x_{i}^{k+1}=\frac{1}{\bar{\lambda}^{k}}\left[\mu^{k}-\left.\left(\frac{\partial f / \partial x_{i}}{\partial h / \partial x_{i}}\right)\right|_{x_{i}=x^{k}}\right]^{n} x_{i}^{k}, & \left(\text { for } \underline{x}_{i}<x_{i}^{k+1}<\bar{x}_{i}\right),
\end{aligned}
$$

where $x_{i}^{k}$ is the $i$ th design variable in the $k$ th iteration, $x_{i}^{k+1}$ the updated size design variable, $f$ the object function, $h$ the constraint function, $\tilde{\lambda}^{k}$ the $\eta$ th power of the Lagrange multiplier in the $k$ th iteration, and $\eta$ a given step parameter. Here $\mu^{k}$ is the shift parameter in the $k$ th iteration, which satisfies

$$
\begin{aligned}
\mu^{k} \geqslant & {\left.\left[\frac{\partial f}{\partial x_{i}} / \frac{\partial h}{\partial x_{i}}\right]\right|_{x_{i}=x_{i}^{k}} } \\
& \quad\left(\text { for } \underline{x}_{i}<x_{i}^{k+1}<\bar{x}_{i} ; i=1,2, \ldots, N\right),
\end{aligned}
$$

where, $N=2 n_{e t}$.

The optimization algorithm based on this updating rule has been sucessfully applied to solve the frequency response optimization problem ${ }^{19}$ and the structural eigenfrequency optimization problem discussed in Ma et al. ${ }^{18}$

\subsection{Updating the orientational design variables}

In order to determine the updating scheme for the orientational design variable, we shall describe an approach given as follows.

The first we shall find the orientations, $\theta_{e}\left(-\pi / 2 \leqslant \theta_{e} \leqslant \pi / 2, e=1,2, \ldots, n_{e l}\right)$, which make the objective function to be maximal (for the problem 1 and 2) or minimal (for the problem 3). Since the Kuhn-Tucker condition with respect to an orientational design variable can be written as:

$$
\frac{\partial \lambda^{*}}{\partial \theta_{e}}=0 \quad\left(-\pi / 2 \leqslant \theta_{e} \leqslant \pi / 2\right),
$$

it follows from Eq. (16) that

$$
\begin{aligned}
g_{e}^{1} \cos 2 \theta_{e} & -g_{e}^{2} \sin 2 \theta_{e}+g_{e}^{3} \cos 4 \theta_{e} \\
& -g_{e}^{4} \sin 4 \theta_{e^{e}}=0\left(-\pi / 2 \leqslant \theta_{e} \leqslant \pi / 2\right) .
\end{aligned}
$$

Equation (24) can also be transformed to a polynomial equation by assuming $z=\operatorname{tg} \theta_{e}$,

$$
b_{4} z^{4}+b_{3} z^{3}+b_{2} z^{2}+b_{1} z+b_{0}=0 .
$$

Note that there may be several solutions satisfying Eqs (23) and (24). In order to determine a unique solution, we defined an evaluation function: ${ }^{20}$

$$
\begin{aligned}
& \sigma_{e}\left(\theta_{e}\right)=g_{e}^{1} \sin 2 \theta_{e}+g_{e}^{2} \cos 2 \theta_{e} \\
& \quad+g_{e}^{3} \sin 2 \theta_{e} \cos 2 \theta_{e}-g_{e}^{4} \sin ^{2} 2 \theta_{e} .
\end{aligned}
$$

By comparing the values of the evaluation function with respect to all the solutions of Eq. (23) and $\theta_{r}= \pm \pi / 2$, respectively, a unique solution, which makes the objective function to be optimal, can be determined. ${ }^{20}$

\section{EXAMPLES}

Several examples of the above stated three kinds of eigenfrequency optimization are described to demonstrate the feasibility of the approach presented in this paper. To this end we shall compare the results with those obtained by using a conventional beam model without changing the topology of the initial structure.

\subsection{Results of different power $\mathrm{n}$ in the mean-eigen- value}

As shown in Fig. 2, a narrow beam-like rectangular design domain is specified: $14.0 \mathrm{~cm}$ in horizontal length and $2.0 \mathrm{~cm}$ in vertical height with two fixed supported boundaries at the left and right ends with a concentrated mass at the center of domain. Young's modulus is given as $100 \mathrm{~kg} \mathrm{~cm}^{-2}$, the mass density $\rho_{0}=10^{-6} \mathrm{~kg} \mathrm{~cm}^{-3}$, and the concentrated mass $5.0 \times 10^{-6} \mathrm{~kg} \mathrm{~cm}^{-3}$. The finite-element model is defined by $140 \times 204$ node quadrilateral plane stress elements with 2961 nodes. The constraint of total mass is given by $V_{0}=9.0 \times 10^{-6} \mathrm{~kg} \mathrm{~cm}^{-3}$. The initial design is assumed to be uniformly perforated as $a_{e}=b_{e}=0.176$, so that the total mass constraint is nearly satisfied.

To investigate the effect of power $n$ in the meaneigenvalue, we consider the problems maximizing the mean-eigenvalue with respect to different values of $n$, i.e. $n=-2,-1,0,1,2$. Here, the lowest three eigenfrequencies of the structure are used to define the mean-eigenvalue, and $w_{i}=1.0(i=1,2,3)$ are assumed with $\lambda_{0_{t}}=0.0(i=1,2,3)$.

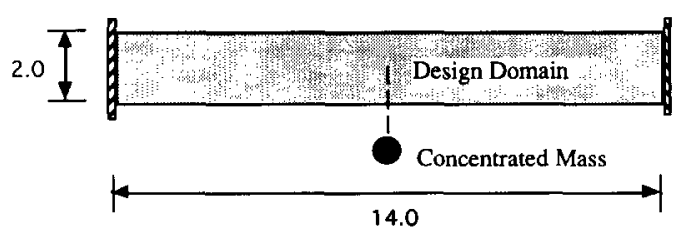

Fig. 2. Design domain of the structure. 
Table 1. Eigenfrequencies at the optimized structure with respect to the different $n$

\begin{tabular}{ccrrrrr}
\hline Frequency & Initial & $n=2$ & $n=1$ & $n=0$ & $n=-1$ & $n=-2$ \\
\hline$\omega_{1}$ & 16.3 & 21.0 & 29.8 & 53.2 & 62.2 & 63.2 \\
$\omega_{2}$ & 51.6 & 53.6 & 110.3 & 139.2 & 134.2 & 124.8 \\
$\omega_{3}$ & 63.8 & 239.3 & 244.9 & 202.2 & 156.3 & 162.7 \\
Max. $+\%$ & & $275 \%$ & $284 \%$ & $226 \%$ & $282 \%$ & $287 \%$ \\
\hline
\end{tabular}

Table 1 shows the eigenfrequencies of the initial and optimal structures obtained by the method presented in this paper, while Fig. 3 shows the optimal configuration (layout) of structures. As shown in
Table 1, when $n=0, n=-1$ and $n=-2$, the lowest eigenfrequency $\omega_{1}$ has the biggest improvement in this optimization. On the other hand, when $n=1$ and $n=2$, the highest eigenfrequency $\omega_{3}$ in three chosen

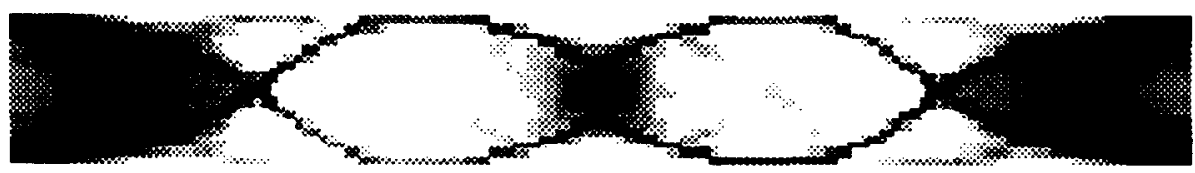

(a) $n=2$

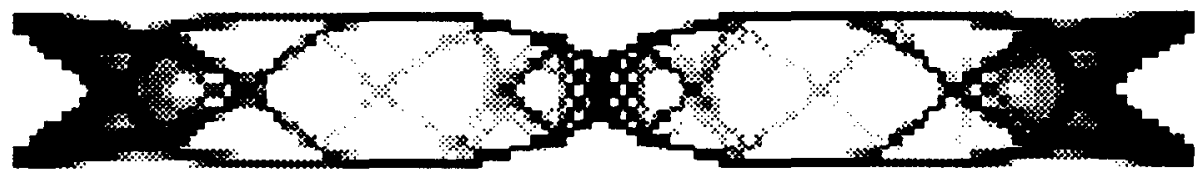

(b) $n=1$

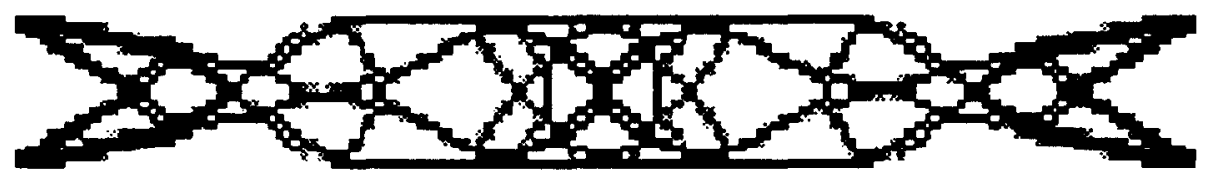

(c) $n=0$

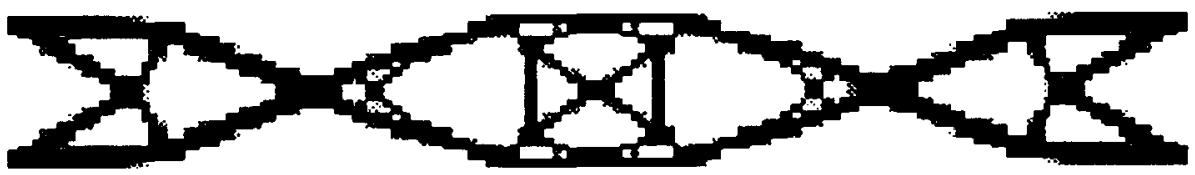

(d) $n=-1$

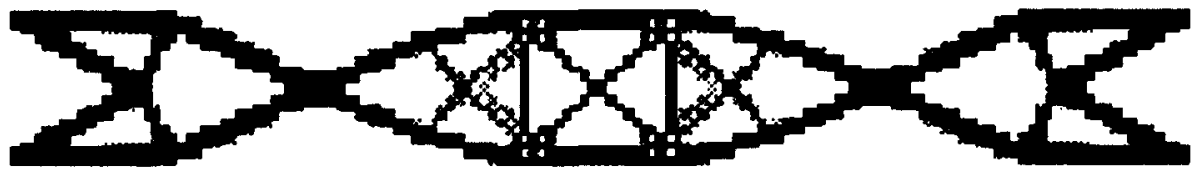

(e) $\mathbf{n}=-2$

Fig. 3. Optimal structures with respect to different values of $n$ for the microstructural model: (a) $n=2$ : (b) $n=1$; (c) $n=0$; (d) $n=-1$; and (e) $n=-2$. 
Table 2. Optimized eigenfrequencies with respect to the different values of $n$ for the beam model

\begin{tabular}{crrrrrr}
\hline Frequency & Initial & $n=2$ & $n=1$ & $n=0$ & $n=-1$ & $n=-2$ \\
\hline$\omega_{1}$ & 21.6 & 10.8 & 11.1 & 25.2 & 28.2 & 28.3 \\
$\omega_{2}$ & 92.8 & 43.0 & 44.6 & 111.6 & 100.4 & 96.4 \\
$\omega_{3}$ & 149.4 & 279.7 & 280.2 & 209.4 & 177.1 & 171.9 \\
Max.+\% & & $87 \%$ & $88 \%$ & $17 \%$ & $31 \%$ & $31 \%$ \\
\hline
\end{tabular}

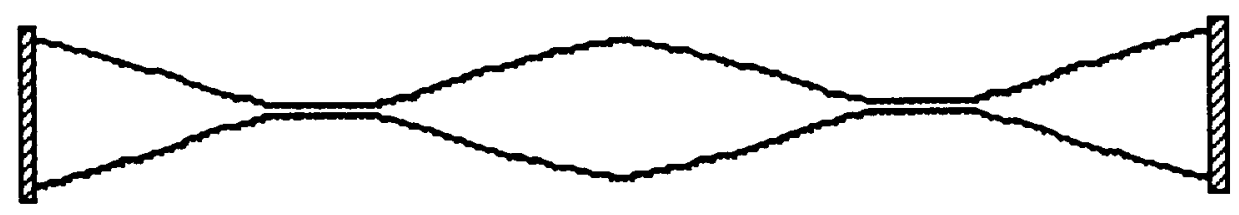

(a) $n=2$

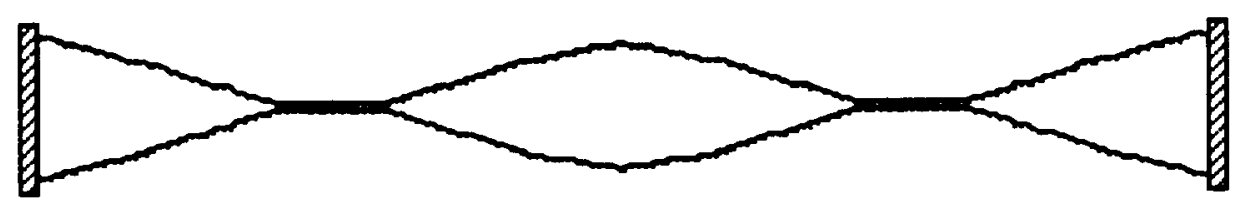

(b) $n=1$

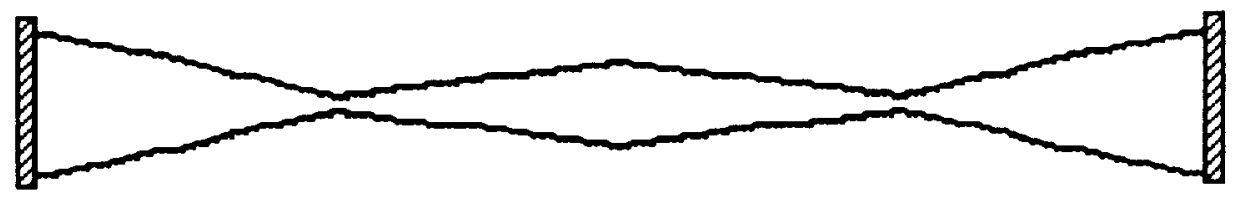

(c) $n=0$

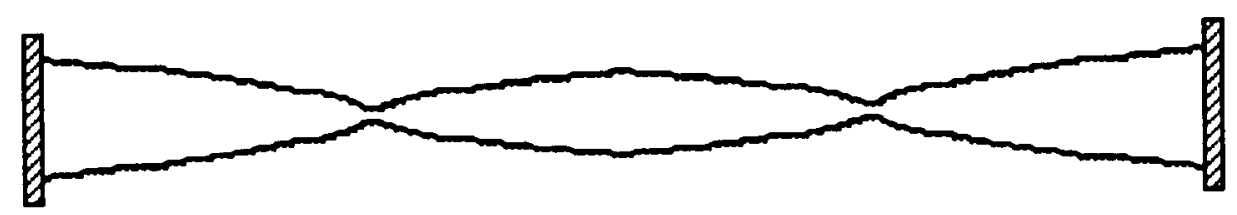

(d) $n=-1$

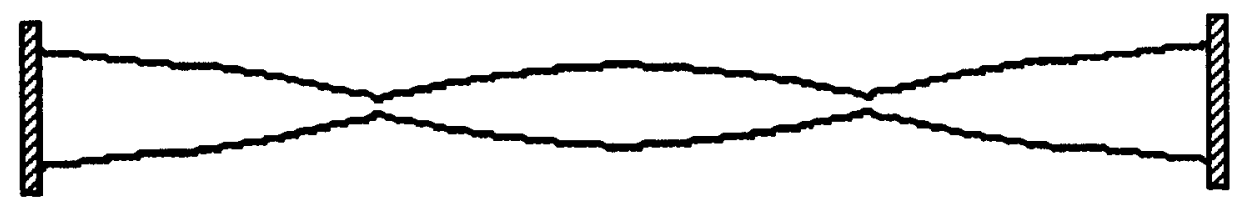

(e) $n=-2$

Fig. 4. Optimal structures with respect to different values of $n$ for the beam model: (a) $n=2$; (b) $n=1$ (c) $n=0$; (d) $n=-1$; and (e) $n=-2$. 


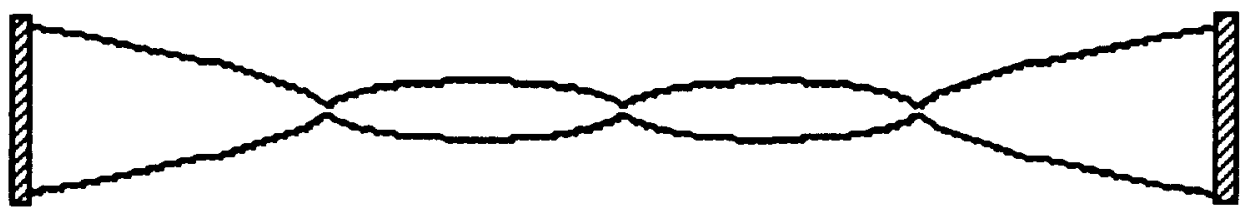

(a) $\omega_{0}=80 \mathrm{~Hz}$

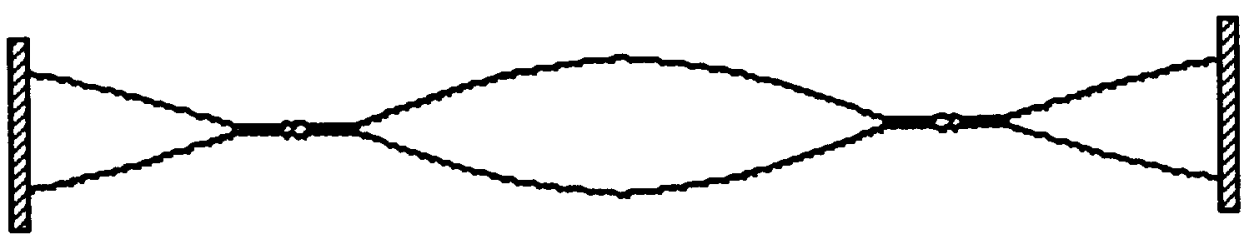

(b) $\omega_{0}=150 \mathrm{~Hz}$

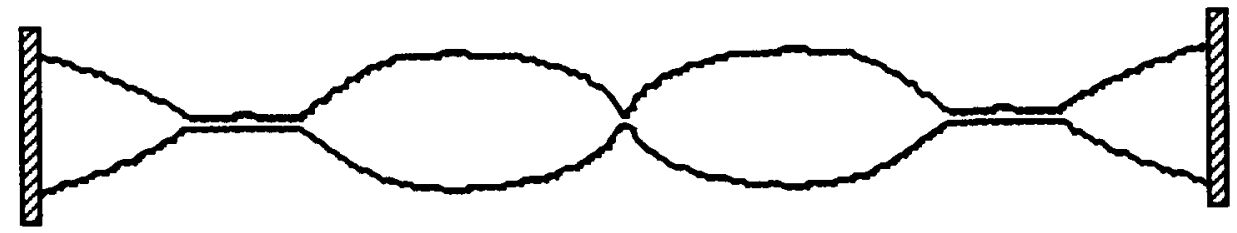

(c) $\omega_{0}=200 \mathrm{~Hz}$

Fig. 5. Maximization of gaps between structural eigenfrequencies and a given frequency: (a) $\omega_{0}=80 \mathrm{~Hz}$; (b) $\omega_{0}=150 \mathrm{~Hz}$; and (c) $\omega_{0}=200 \mathrm{~Hz}$.

eigenfrequencies has the biggest improvements. It has been mentioned previously that when $n \leqslant 0$, the lowest eigenfrequency has the largest contribution to the mean-eigenvalue, thus the problem becomes mainly to maximize the lowest eigenfrequency; conversely the highest eigenfrequency has the largest contribution to the mean-eigenvalue, thus the problem becomes mainly to maximize the highest eigenfrequency. Table 1 also shows that a $287 \%$ improvement can be obtained for the lowest eigenfrequency, while $283 \%$ for the highest eigenfrequency. Note that, as shown in Fig. 3, the optimal structure becomes stiffer and stiffer along with the decreasing of power $n$, this is because that the lowest eigenfrequency has a larger and larger contribution to the mean-eigenvalue when the power $n$ is decreasing. Therefore, for obtaining a stiffer structure, $n$ should be chosen as a non-positive number.
To compare the above results, a conventional beam model is developed and is optimized using the size variables. Here 224 beam elements with 225 nodes are used to define a finite-element beam model. The objective function, volume constraint, Young's modulus and mass density are all the same as the problem discussed above. Table 2 gives the eigenfrequencies of the initial and final structures of the beam model. As shown in Table 2, the same tendency is obtained as the topology and shape optimization. However, the maximal improvement in the lowest eigenfrequency is only $31 \%$, and in the highest eigenfrequency $88 \%$. It is only one-tenth (for the lowest eigenfrequency) or one-third (for the highest eigenfrequency) of that obtained by changing the topology and shape. Therefore, using the topology and shape optimization method presented in this paper can greatly improve the design.

Table 3. Eigenfrequency results for maximizing gaps using the beam model

\begin{tabular}{ccccc}
\hline Frequency & Initial & $\omega_{0}=80 \mathrm{~Hz}$ & $\omega_{0}=150 \mathrm{~Hz}$ & $\omega_{0}=200 \mathrm{~Hz}$ \\
\hline$\omega_{1}$ & 21.6 & 8.95 & 9.67 & 5.51 \\
$\omega_{2}$ & 92.8 & 124.6 & 35.9 & 32.4 \\
$\omega_{3}$ & 149.4 & 138.6 & 271.4 & 40.0 \\
$\omega_{4}$ & 300.8 & 280.5 & 298.3 & 399.8 \\
\hline
\end{tabular}




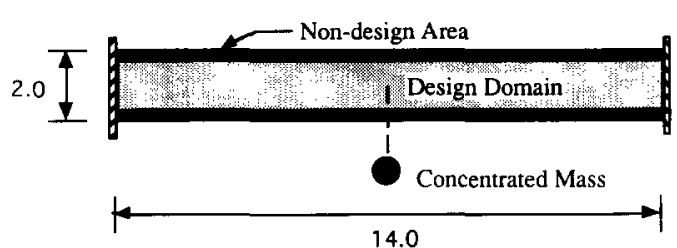

Fig. 6. Reinforcement optimization problem.

Figure 4 show the optimal structures obtained using the sizing optimization. Comparing Fig. 4 with Fig. 3, both the topology and shape optimization and the sizing optimization obtain the similar shapes of the outside in the cases of $n<0$. However, every different structures were obtained in the case $n>0$, since higher modes of two different models are not comparable.

\subsection{Examples of maximizing the gaps between the frequencies}

First the conventional beam for the sizing optimization, then the microstructure model for the reinforcement optimization are used to demonstrate the second kind optimization problem that maximizes the gaps between the specified structural eigenfrequencies and the given frequencies $\omega_{0_{i}}(i=1,2, \ldots, m)$.

The beam model used here is the same with that in the previous example except the shift parameters $\omega_{0},(i=1,2, \ldots, m)$. Here they are assumed not to be zeros. Three cases are calculated for this example, with respect to the different parameters (the different given frequencies): (a) $\omega_{0}=80 \mathrm{~Hz}$, (b) $\omega_{0}=150 \mathrm{~Hz}$; and (c) $\omega_{0}=200 \mathrm{~Hz}$, where, $\omega_{0_{1}}=\omega_{0}$ $(i=1,2, \ldots, m)$. In case (a) the 1 st and 2 nd modes were used, i.e. $m=2$; in the case (b) $m=3$; in case (c) $m=4$. It is also assumed to be $n=-2$.

Figure 5 shows the final shape of the beam structure, while Table 3 shows the eigenfrequencies obtained. As shown in Table 3, the maximal gap obtained is between the 1st and 2nd eigenfrequencies
Table 4. Eigenfrequency results of the reinforcement optimization

\begin{tabular}{crrc}
\hline Frequency & Initial & $n=-1$ & $n=-2$ \\
\hline$\omega_{1}$ & 21.6 & 62.2 & 27.7 \\
$\omega_{2}$ & 62.5 & 207.0 & 215.1 \\
$\omega_{3}$ & 81.7 & 216.8 & 215.1 \\
$\omega_{4}$ & 89.3 & 279.7 & 237.8 \\
$\omega_{5}$ & 151.2 & 410.6 & 250.2 \\
\hline
\end{tabular}

when $\omega_{0}=80 \mathrm{~Hz}$; between 2nd and 3rd eigenfrequencies when $\omega_{0}=150 \mathrm{~Hz}$; between the $3 \mathrm{rd}$ and 4th eigenfrequencies when $\omega_{0}=200 \mathrm{~Hz}$.

For the reinforcement optimization problem, the design domain is the same as in Fig. 2, but a core-structure as a non-design area is specified as shown in Fig. 6. Here, the lowest five eigenfrequencies are used in the optimization problem, and the given eigenfrequency $\omega_{0}$ is assumed to be $100 \mathrm{~Hz}$, where $\omega_{0_{i}}=\omega_{0}(i=1,2, \ldots, 5)$.

Two cases with the different power $n$, (a) $n=-1$; and (b) $n=-2$, are considered. Figure 7 shows the optimal reinforcements obtained by the method presented in this paper. Table 4 shows the eigenfrequency result. As shown in Table 4, in the both cases, the maximal gap is between the 1st and 2nd eigenfrequencies. In the case (a), the first eigenfrequency has a large undesirable increment. Conversely the first eigenfrequency only has a small increment in the case (b). The maximal gap between the 1st and 2nd eigenfrequencies can be improved $29 \%$ by use of an even number for $n$ comparing with the use of an odd number.

\subsection{Examples of obtaining prescribed eigenfrequen- cies}

In order to obtain the prescribed desired structural eigenfrequencies, the parameters $\omega_{0_{i}}(i=1,2, \ldots, m)$ are given in the desired eigenfrequencies, and the power $n$ is given as a positive even number, i.e. $n=2$. Moreover, the weighting coefficients are chosen as

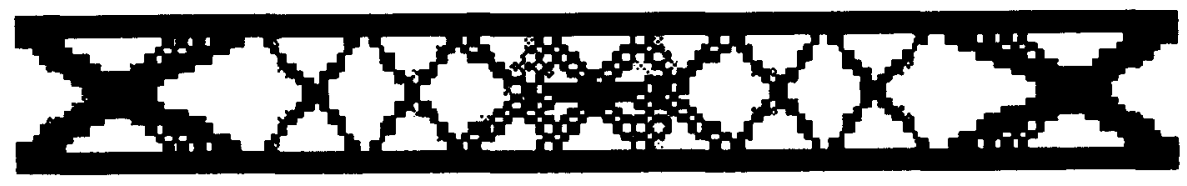

(a) $n=-1$

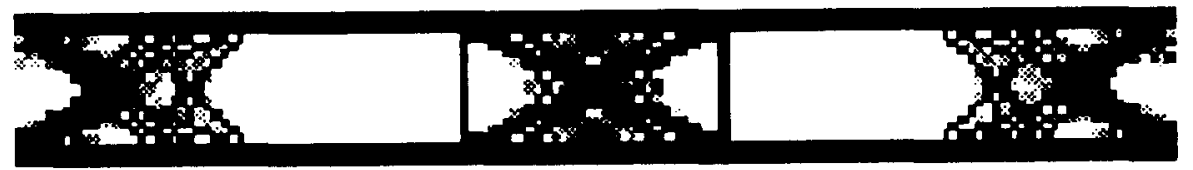

(b) $n=-2$

Fig. 7. Optimal reinforcements: (a) $n=-1$; and (b) $n=-2$. 
Table 5. Desired eigenfrequencies and obtained eigenfrequencies for beam model

\begin{tabular}{crcccc}
\hline & & & & \multicolumn{2}{c}{ (b) } \\
Frequency & Initial & Desired & Obtained & Desired & Obtained \\
\hline$\omega_{1}$ & 21.5 & 25.0 & 24.8 & 65.0 & 12.4 \\
$\omega_{2}$ & 92.8 & 120.0 & 118.5 & 75.0 & 71.7 \\
$\omega_{3}$ & 149.7 & 190.0 & 189.4 & 110.0 & 115.9 \\
\hline
\end{tabular}

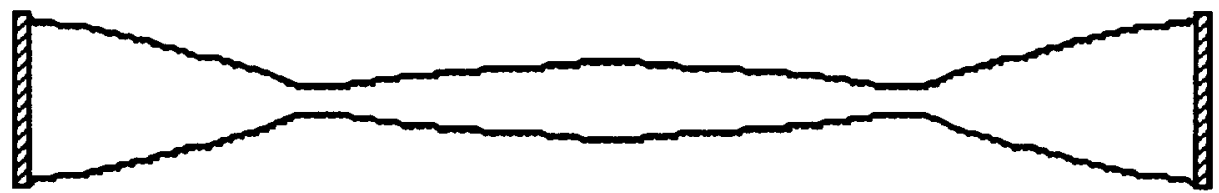

a) Desired eigenfrequencies: $25.0,120.0,190.0 \mathrm{~Hz}$

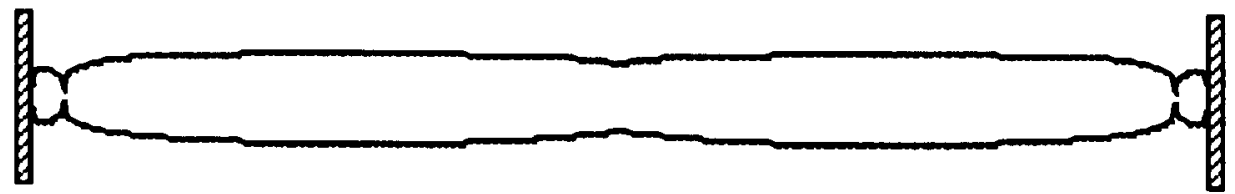

(b) Desired eigenfrequencies: 65.0,75.0,110.0 Hz

Fig. 8. Optimal structure for beam model. Desired eigenfrequencies: (a) 25,120 and $190 \mathrm{~Hz}$; and (b) 65 , 75 and $110 \mathrm{~Hz}$.

$w_{i}=1 / \lambda_{0_{i}}(i=1,2, \ldots, m)$. This is to yield a dimensionless object, and then all eigenfrequencies in the mean-eigenvalue will be able to have equal rates to reach their desired values.

For this example, first, the conventional beam model is considered using the lowest three modes. As shown in Table 5, the three lowest eigenfrequencies of the initial beam are $\omega_{1}=21.5 \mathrm{~Hz}, \omega_{2}=92.8 \mathrm{~Hz}$ and $\omega_{3}=149.7 \mathrm{~Hz}$. Figure 9(a) shows the final structure obtained for the desired eigenfrequencies $\omega_{1}=25 \mathrm{~Hz}, \omega_{2}=120 \mathrm{~Hz}$ and $\omega_{3}=190 \mathrm{~Hz}$ (case a). Figure 9(b) shows the final structure obtained for the desired eigenfrequencies $\omega_{1}=65 \mathrm{~Hz}, \omega_{2}=75 \mathrm{~Hz}$ and $\omega_{3}=110 \mathrm{~Hz}$ (case b). As shown in Table 5, the maximum error between the desired eigenfrequencies and obtained eigenfrequencies is less than $1.3 \%$ for the case (a), but near $80 \%$ for the case (b). It implies that large changes in the structural eigenfrequencies can not be expected for a sizing optimization problem.

Table 6 shows the results using the microstructure model for the same desired eigenfrequencies given in the case (b) of the beam model. The initial structure
Table 6. Desired eigenfrequencies and obtained eigenfrequencies for microstructure model

\begin{tabular}{cccc}
\hline Frequency & Initial & Desired & Obtained \\
\hline$\omega_{1}$ & 16.3 & 65.0 & 63.5 \\
$\omega_{2}$ & 51.6 & 75.0 & 74.1 \\
$\omega_{3}$ & 63.8 & 110.0 & 110.4 \\
\hline
\end{tabular}

has the eigenfrequencies $\omega_{1}=16.3 \mathrm{~Hz}, \omega_{2}=51.6 \mathrm{~Hz}$ and $\omega_{3}=63.8 \mathrm{~Hz}$. As shown in the Table 6 , the final eigenfrequencies obtained are $\omega_{1}=63.5 \mathrm{~Hz}$, $\omega_{2}=74.1 \mathrm{~Hz}$ and $\omega_{3}=110.4 \mathrm{~Hz}$. The maximum error between the desired eigenfrequencies and computed eigenfrequencies is less than $2.4 \%$. Therefore, the use of the microstructure model makes it possible to obtain the desired eigenfrequencies which have larger changes from the initial ones. Figure 9 shows the final configuration obtained by using the microstructure model.

Another example for the desired eigenfrequency problem is also calculated in this paper. The purpose is to design a plane bridge-like structure for the prescribed structural eigenfrequencies. The design

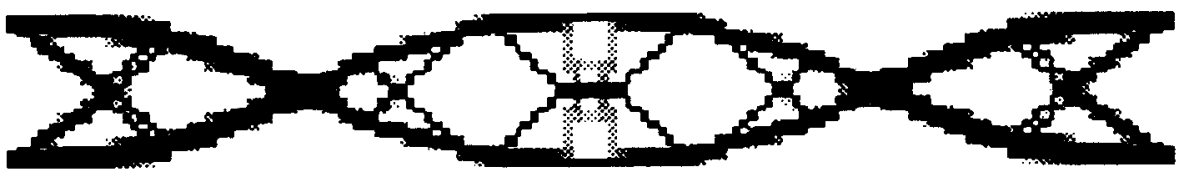

Fig. 9. Optimal structure for the microstructure model (desired eigenfrequencies: $65.0,75.0,110.0 \mathrm{~Hz}$ ). 


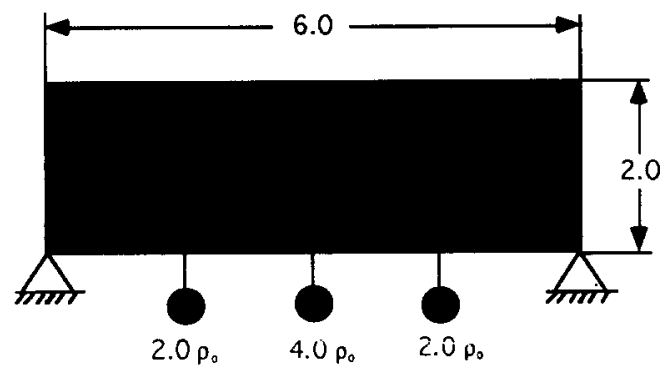

(a) Design domain and loading

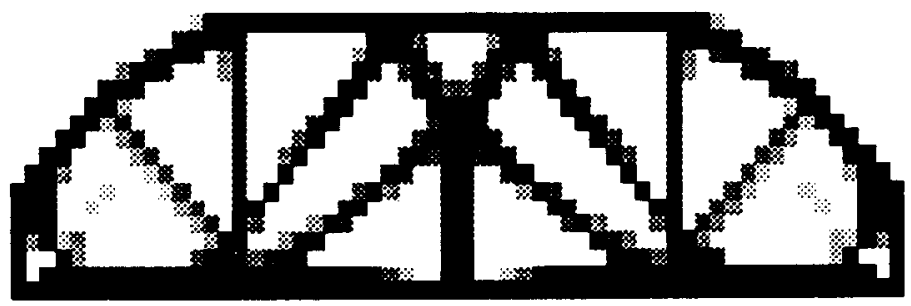

(b) Case I

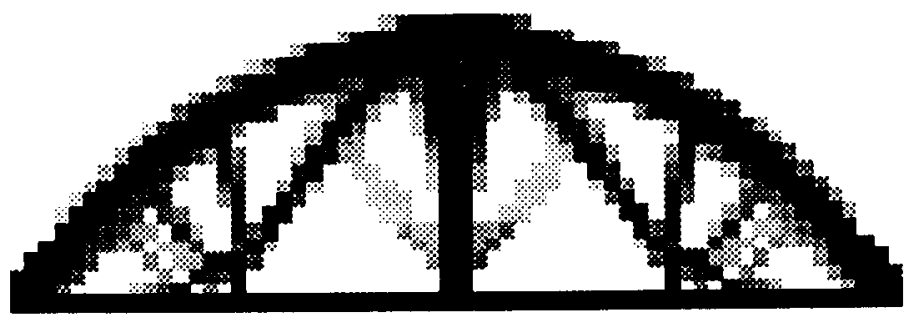

\section{(c) Case II}

Fig. 10. Optimal topologies for the bridge-like structure: (a) design domain and loading; (b) Case I; and (c) Case II.

domain and boundary conditions are given in Fig. 10(a). The loading is assumed by the three concentrated masses, $2.0 \rho_{0}, 4.0 \rho_{0}$ and $2.0 \rho_{0}$ on the bottom of bridges as shown in Fig. 10(a). Two cases with respect to different groups of desired eigenfrequencies are calculated. Figures $10(\mathrm{~b})$ and 10(c) describe the final structures. Table 7 gives the comparison between the initial eigenfrequencies, the desired eigenfrequencies and the final eigenfrequencies obtained. It is shown that the use of the method presented in this paper makes it possible to obtain the desired eigenfrequencies with quite good accuracy.

\section{CONCLUSIONS}

A mean-eigenvalue is defined and three kinds of optimization problems are discussed to obtain desired eigenfrequencies of a structure. The topology and shape optimization technique using the concept of OMD is utilized to determine the optimum. The sensitivity analysis of the mean-eigenvalue is also given. An improved updating algorithm is then applied for solving the optimization problem. Several examples are given to demonstrate the feasibility of the approach presented in this paper. It has been shown that the method presented in this paper is

Table 7. Desired eigenfrequencies and obtained eigenfrequencies for the bridge-like structure

\begin{tabular}{|c|c|c|c|c|c|}
\hline \multirow[b]{2}{*}{ Frequency } & \multirow[b]{2}{*}{ Initial } & \multicolumn{2}{|c|}{ Case I } & \multicolumn{2}{|c|}{ Case II } \\
\hline & & Desired & Obtained & Desired & Obtained \\
\hline$\omega_{1}$ & 21.5 & 80.0 & 79.5 & 110.0 & 108.7 \\
\hline$\omega_{2}$ & 34.7 & 170.0 & 169.2 & 130.0 & 128.4 \\
\hline$\omega_{3}$ & 49.9 & 200.0 & 199.9 & 170.0 & 170.9 \\
\hline
\end{tabular}


applicable to various eigenfrequency optimization problems in structural design, and makes it possible to obtain a truly optimal structures, which is optimal in the size, shape and topology at once.

Acknowledgments - This research is supported by the NISSAN Co., LTD, and the authors are grateful for this support.

\section{REFERENCES}

1. V. B. Venkayya, "Structural optimization review and some recommendations," International Journal for $\mathrm{Nu}$ merical Methods in Engineering 13, 203-228 (1978).

2. N. Olhoff, and J. E. Taylor, "On structural optimization," Journal of Applied Mechanics 50, 1139-1151 (1983).

3. R. T. Haftka and R. V. Grandhi, "Structural shape optimization-a survey," Computer Methods in Applied Mechanics and Engineering 57, 91-106 (1986).

4. B. L. Pierson, "A survey of optimal structural design under dynamic constraints," International Journal for Numerical Methods in Engineering 4, 491-499 (1972).

5. R. V. Grandhi and V. B. Venkayya, "Structural optimization with frequency constraints," AIAA Journal 26, $858-866(1988)$

6. T.-Y. Chen, "Optimum design of structures with both natural frequency and frequency response constraints," International Journal for Numerical Methods in Engineering 33, 1927-1940 (1992).

7. N. S. Khot, "Optimization of structures with multiple frequency constraints," Computers \& Structures 20, 869-876 (1985)

8. F. I. Niordson, "On the optimal design of a vibrating beam," Quarterly. Journal of Applied Mathematics 23, $47-53$ (1965).

9. R. M. Brach, "On the extremal fundamental frequencies of vibrating beams." International Journal of Solids and Structures 4, 667-674 (1968).
10. W. Prager and J. E. Taylor, "Problems of optimal structural design," Journal of Applied Mechanical Trans. Am. Soc. Mech. Engrs 90, 102-106 (1968).

11. M. S. Zarghamee, "Optimum frequency of structures." AIAA Journal 6, 749-750 (1968).

12. C. A. Prato, "Maximization of eigenvalues by conjugate gradients," Journal of Structures Div. Am. Soc. Civ. Engrs 96, 171-176 (1970).

13. N. Olhoff, "Optimization of vibrating beams with respect to higher order natural frequencies," Journal of Structural Mechanics 4, 87-122 (1974).

14. M. P. Bendsøe and N. Olhoff, "A method of design against vibration resonance of beams and shafts," Optimal Control Applications \& Methods 6, 191-200 (1985).

15. N. Olhoff, "Structural optimization by variational method," in Computer Aided Optimal Design: Structural and Mechanical Systems (edited by C. A. Mota Soares), pp. 87-164. Springer-Verlag, 1986.

16. M. P. Bendsøe and N. Kikuchi, "Generating optimal topologies in structural design using a homogenization method," Computational Methods in Applied Mechanical Engineering 71, 197-24 (1988).

17. A. Diaz and N. Kikuchi, "Solution to shape and topology eigenvalue optimization problems using a homogenization method," International Journal for $\mathrm{Nu}$ merical Methods in Engineering 35, 1487-1502 (1992).

18. Z.-D. Ma, H.-C. Cheng and N. Kikuchi, "Topography and shape optimization technique for structural dynamic problems," Recent Advances in Structural Problems, ASME, PVP-Vol. 248/NE-Vol. 10, pp. 133-143 (1992).

19. Z.-D. Ma,. N. Kikuchi and Y. Hagiwara, "Structural topology and shape optimization for a frequency response problem," Computational Mechanics, 13(3), 157-174 (1993).

20. H.-C. Cheng, Z.-D. Ma and N. Kikuchi, "An improved approach for obtaining the optimal orientation of orthotropic material," preprint, Department of Mechanical Engineering and Applied Mechanics, The University of Michigan, Ann Arbor, MI, 48109.2215, 1993. 